\title{
Analgesic activity of high-dose intravenous calcitonin in cancer patients with bone metastases
}

\author{
NICOLAS TSAVARIS ${ }^{1}$, PETROS KOPTERIDES ${ }^{1}$, CHRISTOS KOSMAS $^{2}$, MARIA VADIAKA $^{1}$, \\ ANTONIOS DIMITRAKOPOULOS ${ }^{1}$, HELIAS SCOPELITIS ${ }^{1}$, ROXANNI TENTA ${ }^{1}$, \\ GEORGE VAIOPOULOS ${ }^{1}$ and CHRISTOS KOUFOS ${ }^{1}$ \\ ${ }^{1}$ Department of Pathophysiology, Oncology Unit, 'Laiko' General Hospital, University of Athens \\ School of Medicine, Athens; ${ }^{2}$ 2nd Department of Oncology, 'Metaxa' Hospital, Piraeus, Greece
}

Received January 24, 2006; Accepted April 17, 2006

\begin{abstract}
We undertook a prospective, nonrandomized study with the objective to evaluate the efficacy of salmon calcitonin (sCT) in controlling pain secondary to bone metastases. Our study population consisted of 45 cancer patients with bone metastases (26 men) with a mean age of 64 years (range, 4870) who had completed chemotherapy, hormonal therapy and radiation therapy at least 30 days prior to enrollment in the study, and had intractable pain despite the use of common analgesics (acetaminophen, nonsteroidal anti-inflammatory agents, opioids) and bisphosphonates. The study medication was a 300-IU dose of sCT administered intravenously daily for 5 consecutive days and repeated every two weeks until no response was noticeable. The analgesic efficacy of SCT was evaluated by means of Huskisson's visual analogue scale and Keele's pain scale; the daily consumption of analgesic drugs and performance status were also monitored. None of the patients managed to completely discontinue the use of other analgesics, but 5 patients (11\% of the total number) had an analgesic response that lasted 4 weeks and less than $5 \%$ of the patients continued to respond for 6 weeks. No significant side effects were observed. Our data show that intravenous calcitonin administered in a relatively high dose has a very limited therapeutic potential as an adjuvant analgesic for a short period of time in selected cancer patients with bone metastases.
\end{abstract}

\section{Introduction}

Bone pain remains the most common cause of cancer-related pain and its intensity depends on the site of the tumor and

Correspondence to: Dr Petros Kopterides, Department of Pathophysiology, Oncology Unit, 'Laiko' General Hospital, University of Athens School of Medicine, 28-30 Korai Street, Nea Ionia, Athens 14233, Greece

E-mail: petkop@ath.forthnet.gr

Key words: bone metastases, calcitonin, cancer, pain, palliative care, analgesia, opioids, bisphosphonates progression of the underlying disease (1). Primary malignant bone tumors are relatively uncommon but approximately $80 \%$ of patients with breast, lung and prostate cancer develop bone metastases (2). More than half of these patients suffer from pain and functional disability and approximately $20 \%$ experience a bone fracture and/or hypercalcemia (3). The fact that approximately 1 in 3 individuals in the developed world develops cancer and almost half of them die of progressive disease highlights the magnitude of the problem of metastatic bone disease (4).

Multiple modalities are used nowadays to manage metastatic bone pain. Some of them target the tumor itself (e.g. surgery, chemotherapy, radiotherapy and hormonal manipulation) while others target the pain [e.g. acetaminophen, nonsteroidal anti-inflammatory drugs (NSAIDs), bisphosphonates and opioids]. Unfortunately, the use of conventional analgesics alone or in combination does not always produce a satisfactory analgesic result and may be complicated by undesirable side effects. Therefore, our armamentarium against metastatic bone pain is not complete.

Calcitonin has potential efficacy in reducing pain and improving bone density but its current use for the relief of metastatic bone pain is not very common; characteristically, while it is mentioned in one recent review dealing with the issue of cancer pain (5), another makes no reference to it (6). Mostly low doses (100-200 IU) of sCT have been used in the management of metastatic bone pain $(7,8)$. It is also noteworthy that the last clinical study assessing the efficacy of calcitonin in the management of cancer pain was published more than 5 years ago (9). Therefore, its role as an analgesic has not been clarified to satisfaction.

We studied the analgesic activity of a high-dose (300 units) of intravenous salmon calcitonin ( $\mathrm{SCT}$ ) in 45 patients with bone metastases who suffered from pain, whose treatment with common analgesics (acetaminophen, NSAIDs, opioids) and bisphosphonates had failed, and who had completed their radiotherapy and chemotherapy treatment courses.

\section{Materials and methods}

Inclusion and exclusion criteria. Patients were eligible for inclusion in the study if they suffered from metastatic bone 
Table I. Patients' demographics and characteristics.

\begin{tabular}{cc}
\hline Parameter & $\begin{array}{c}\text { Number of } \\
\text { patients }\end{array}$ \\
\hline
\end{tabular}

Demographics

Age [mean (range)] in years

Gender

Male

Female

Type of cancer

Breast
Lung (non-small cell)
Lung (small-cell)
Prostate
Cervix

Sarcoma

Performance status (Karnofsky index: 60/70)

Previous chemotherapy

Previous hormonal therapy

Previous radiotherapy

Analgesic treatment prior to calcitonin treatment

Fentanyl

Acetaminophen + NSAIDs + morphine ${ }^{a}$

Acetaminophen + codeine/morphine ${ }^{\mathrm{a}}+$ NSAIDs

Aspirin + codeine/morphine ${ }^{a}$ disorders, and current use of tranquillizers or steroids were excluded. Inability to make a self-evaluation was also considered an exclusion criterion.

Patients. Forty-five patients (26 men) with a mean age of 64 years (range, 48-70) and histologically confirmed cancer with bone metastases (23 with non-small cell lung cancer; 11 with breast cancer; 4 with prostate cancer; 4 with small-cell lung cancer; 2 with cervical cancer, and 1 with sarcoma) entered the study. The Karnofsky performance index was 70 in 16 patients and 60 in 29 patients (Table I).

Study design. After enrollment in the study, patients underwent a complete physical examination and basic laboratory work-up including complete blood count and comprehensive metabolic panel. Side effects were monitored and laboratory work-up was repeated before the administration of each new cycle. No patient received chemotherapy, radiotherapy or hormonal therapy during the study period. Intravenous calcitonin was administered once every two weeks until no response was noticeable.

The study protocol was reviewed and approved by the ethics committee of our hospital. All patients gave written informed consent.

Drug administration. The study medication was a 300-IU dose of calcitonin (Miacalcin; Novartis Pharmaceuticals Corp.) diluted in $250 \mathrm{ml}$ of normal saline and administered intravenously over $60 \mathrm{~min}$, every day, for 5 consecutive days. Therapy was repeated every 2 weeks. Premedication included an intravenous bolus of $100 \mathrm{mg}$ hydrocortisone (Solu-cortef; Pharmacia \& Upjohn) followed by an antihistamine [4 $\mathrm{mg}$ of intravenous dimetindene (Fenistil; Ciba-Geigy)].

Outcome measurement. The primary efficacy end point of our trial was the analgesic response to the study medication as judged from a patient-completed daily pain diary. The intensity of the pain was evaluated by Keele's pain scale and Huskisson's visual analogue scale, before starting therapy and on the 5th, 10th and 14th days after the administration of calcitonin.

We established two stages of analgesic response based on the patients' self-report on their daily analgesic requirements,

Table II. Response to calcitonin administration during the 4 courses of therapy.

\begin{tabular}{|c|c|c|c|c|c|}
\hline & Treatment effect & Day 1 & Day 5 & Day 10 & Day 14 \\
\hline \multirow[t]{2}{*}{1 st course } & No response & 37 & 28 & 36 & 38 \\
\hline & Response & 8 & 17 & 9 & 7 \\
\hline \multirow[t]{2}{*}{ 2nd course } & No response & 0 & 1 & 1 & 2 \\
\hline & Response & 7 & 6 & 6 & 5 \\
\hline \multirow[t]{2}{*}{3 rd course } & No response & 0 & 1 & 1 & 3 \\
\hline & Response & 5 & 4 & 4 & 2 \\
\hline \multirow[t]{2}{*}{ 4th course } & No response & 0 & 1 & 2 & 0 \\
\hline & Response & 2 & 1 & 0 & 0 \\
\hline
\end{tabular}




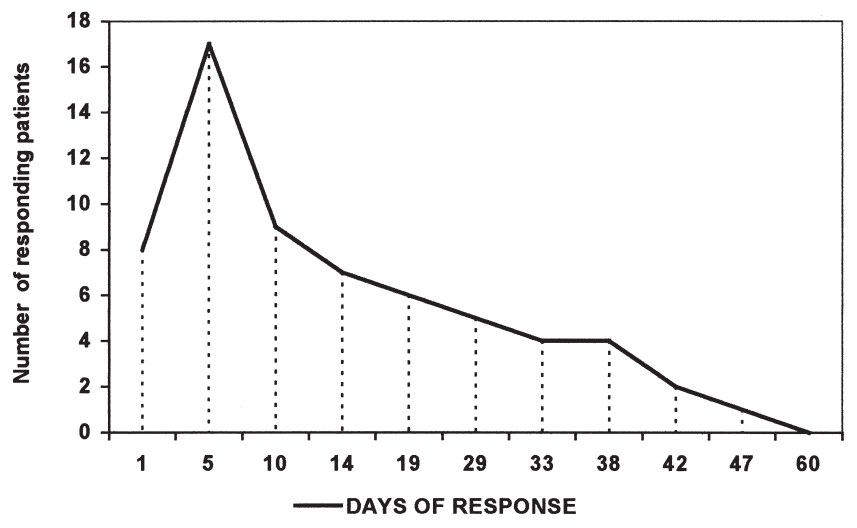

Figure 1. Duration of analgesic response to calcitonin.

pain assessment according to Keele's and Huskisson's scales and changes in their performance status: (i) No response, the pain either remained the same in Keele's and Huskisson's scales or decreased by $<30 \%$, performance status deteriorated and/or analgesic therapy requirements did not change or even increased; (ii) response, there was an improvement of $>30 \%$ in Keele's and Huskisson's scales, performance status improved and/or daily requirements for analgesics decreased.

\section{Results}

Response to calcitonin therapy. Of the 45 patients initially entering the study, 38 (84\%) failed completely by the end of the first cycle and, therefore, received only 1 course of therapy. Accordingly, at the 14th day of the first course, 7 (16\%) patients were still responding and proceeded to a second course. From these 7 patients, 5 (11\% of the total number of patients) responded to the second course and qualified for administration of the third. During the third course, only 2 patients (4\%) showed a response and continued with the fourth course. During the last course, these 2 patients failed to respond (Table II).

None of the patients managed to completely discontinue the use of other analgesics, and none of them continued therapy after the fourth course since all had pain unresponsive to calcitonin.

Time course of response to calcitonin administration - side effects. The main analgesic effect of calcitonin appeared during the first 5 days and, secondarily, the next 10 days (Fig. 1). After this time, the analgesic effect progressively waned, with the longer period of monitored response being approximately 45 days in a small percentage of patients $(<4 \%)$. No side effects necessitating withdrawal from the study were observed.

\section{Discussion}

Despite the implementation of systemic modalities, such as chemotherapy, hormonal therapy, surgery and radiation therapy, and the established role of bisphosphonates, a substantial number of cancer patients still experience pain secondary to bone metastases, which makes the conventional use of analgesics (acetaminophen, NSAIDs, opioids), alone or in combination, necessary. A simple opioid-based regimen will provide successful analgesia in approximately $70 \%$ of patients but, unfortunately, without being free of undesirable side effects.

Calcitonin is a hormone produced in the thyroid gland. Its analgesic activity has been demonstrated in humans (7-13) and animals (14-17), but its mechanism of analgesic action remains unclear. Several hypotheses have been formulated to account for it. Firstly, it inhibits cyclooxygenase, thus lessening the production of prostaglandins and thromboxane A2 in guinea pig lung (18) and humans (19); however, other studies have found opposite results (20). Secondly, it has been shown to increase ACTH, $\beta$-endorphin and cortisol serum levels (21), indicating a possible role in modulation of the anterior pituitary function, but this is challenged in other studies (22). Thirdly, specific binding sites for salmon calcitonin have been found in the brain of animals $(23,24)$ and humans $(25)$, suggesting a central intrinsic analgesic effect; supported by the successful use of calcitonin as a subarachnoid or epidural injection in humans (26). Finally, calcitonin has been shown to potentiate the analgesic effect of opioids in animals (27).

A recent systematic review of randomized, controlled trials (28) showed calcitonin to be effective in the management of acute pain associated with acute osteoporotic vertebral compression fractures, but its use for the relief of metastatic bone pain is not common and even ignored by a recent review on the management of cancer-related pain (6). Therefore, little information is available about its use in this context. However, Martinez et al (3) recently performed a systematic review of all the available data to determine the effectiveness of calcitonin in reducing metastatic bone pain. Unfortunately, only two studies (including only 90 patients in total) had the methodological quality required by the inclusion criteria. Roth et al (8) compared calcitonin (100 IU administered subcutaneously each day for 28 days) versus placebo administered to a group of 40 women with breast cancer and pain from bone metastases that were followed for one month. The analgesic effect of calcitonin was assessed through analgesic consumption, functional capacity, pain duration, and patient pain selfassessment. There was insufficient evidence that calcitonin is effective for total pain reduction. Blomqvist et al (7) compared calcitonin (100 IU administered subcutaneously each day for three months) versus placebo in 50 women with breast cancer and painful bone metastases who were followed for 24 months. No improvement in general performance or bone pain was detected as measured by a visual analogue scale, the daily duration of pain or consumption of analgesic drugs. The systematic review by Martinez et al (3) concluded that the available evidence was limited, did not support the use of calcitonin to control pain from bone metastases and called for new studies to provide additional information. It should be emphasized that this review did not include the randomised double-blind placebo-controlled clinical trial by Hindley et al (11) because a 4-week cut-off was a prerequisite for inclusion in the review, while the latter study was of two-week duration. At the end of the second week, there was an improvement of pain in significantly more patients in the calcitonin group $(5 / 13,38 \%)$ than in the placebo group $(0 / 12)$ (Fisher's exact two-tailed probability test, $\mathrm{P}<0.05)$. 
We hypothesized that one reason for the lack of efficacy of salmon calcitonin in the previous studies was the use of low doses; so, instead of using the 100-IU dose $(7,8)$, we decided to use a 300 -IU intravenous dose that has been previously shown to be efficacious in a study of 34 patients with lung cancer and bone metastases (29). However, our study results are not as optimistic as those reported by Hindley et al (15) and show that high-dose intravenous salmon calcitonin can help in the management of metastatic bone pain and decrease analgesic requirements in only $10 \%$ of patients for a month; the maximum period of recorded response was 45 days and was seen in a small percentage of patients $(4 \%)$. We also showed that it is difficult to achieve complete relief from bone pain with calcitonin, confirming the findings of Blomqvist et al (7); none of our study patients managed to completely stop previous analgesic therapy. Finally, the lack of significant side effects may be attributed to the intravenous route of administration, as nasal administration has been associated with nasal irritation and subcutaneous administration with pain at the injection site. It is also probable that the aggressive premedication regimen consisting of steroids and antihistamines prevented the development of nausea, anorexia and allergic reactions.

Our study has several limitations. First, it was an open, pilot, proof-of-principle study with a small number of patients with heterogeneous malignancies and without a placebo arm, as our main goal was to assess the efficacy, duration of response and safety of high-dose calcitonin. Follow-up lasted two months and measurement of analgesic consumption was based on the patients' self-reports. We also used a rather impractical and expensive dosing schedule of 5-day administration of intravenous calcitonin in a higher than reported dose and, in addition to the difficulty of replicating this in a clinical setting regimen, calcitonin showed very limited therapeutic potential. The discontinuation of the study medication after only two weeks of unsuccessful treatment can be considered a limitation, especially when, in most studies, calcitonin is used for at least $1-3$ months $(7,8)$. Of note, we used a much higher dose than the one used in those studies (300-IU versus 100-IU) and other investigators have used durations of treatment ( 2 weeks) similar to that in our study in order to evaluate the efficacy of calcitonin (11). Without ignoring the cost-containment era in which we practice medicine, we believe it remains to be seen whether a regimen that is suitable for home care and consists of per os steroids, antihistamines and daily high-dose calcitonin (administered intranasally or subcutaneously) can achieve better analgesic results.

In conclusion, our study shows that intravenous calcitonin administered in a relatively high dose has very limited therapeutic potential as an adjuvant analgesic for a short period of time in selected cancer patients with bone metastases, allowing other modalities (e.g. radiation therapy) to take effect and decreasing the need for common analgesics. The abscence of severe side effects and significant drug interactions could make it useful in the management of cancer patients who already receive multiple medications and are, therefore, at increased risk for drug-drug interactions. Further studies are needed to better define the role of intravenous calcitonin in metastatic bone pain.

\section{References}

1. Portenoy RK: Cancer Pain. Epidemiology and syndromes. Cancer 63: 2298-2307, 1989 .

2. Nielsen OS, Munro AJ and Tannock IF: Bone metastases: pathophysiology and management policy. J Clin Oncol 9: 509-524, 1991.

3. Martinez MJ, Roque M, Alonso-Coello P, Catala E, Garcia JL and Ferrandiz M: Calcitonin for metastatic bone pain. Cochrane Database Syst Rev 3: CD003223, 2003.

4. World Health Organization. The World Health Report 1996: Fighting Disease, Fostering Development, Executive Summary. Geneva, Switzerald: World Health Organization.

5. Lussier D, Huskey AG and Portenoy RK: Adjuvant analgesics in cancer pain management. Oncologist 9: 571-591, 2004.

6. Bruera E and Kirn HN: Cancer pain. JAMA 290: 2476-2479, 2003.

7. Blomqvist C, Elomaa I, Porkka L, Karonen SL and LambergAllardt C: Evaluation of salmon calcitonin treatment in bone metastases from breast cancer - a controlled trial. Bone 9: 45-51, 1988.

8. Roth A and Kolaric K: Analgesic activity of calcitonin in patients with painful osteolytic metastases of breast cancer. Results of a controlled randomized study. Oncology 43: 283-287, 1986.

9. Mystakidou K, Befon S, Hondros K, Kouskouni E and Vlahos L: Continous subcutaneous administration of high-dose salmon calcitonin in bone metastasis: pain control and betaendorphin plasma levels. J Pain Symptom Manage 18: 323-330, 1999.

10. Allan E: Calcitonin in the treatment of intractable pain from advanced malignancy. Pharmatherapeutica 3: 482-486, 1983.

11. Hindley AC, Hill EB, Leyland MJ and Wiles AE: A doubleblind controlled trial of salmon calcitonin in pain due to malignancy. Cancer Chemother Pharmacol 9: 71-74, 1982.

12. Serdengecti S, Serdengecti K, Derman U and Berkarda B: Salmon calcitonin in the treatment of bone metastases. Int J Clin Pharmacol Res 6: 151-155, 1986.

13. Szanto J, Ady N and Jozsef S: Pain killing with calcitonin nasal spray in patients with malignant tumors. Oncology 49: 180-182, 1992.

14. Borowicz B, Sagan M, Teter M and Dec-Szlichtyng M: Influence of salmon calcitonin on the analgesic effect of selective kappaopioid agonist in mice. Ann Univ Mariae Curie Sklodowska 56: 407-411, 2001.

15. Martin MI, Goicoechea C, Ormazabal MJ and Alfaro MJ: Effect of the intraperitoneal administration of salmon-calcitonin on the 'in vitro' actions of opioid agonists. Gen Pharmacol 26: 1695-1699, 1995.

16. Umeno H, Nagasawa $\mathrm{T}$, Yamazaki $\mathrm{N}$ and Kuraishi $\mathrm{Y}$ : Antinociceptive effects of repeated systemic injections of calcitonin in formalin-induced hyperalgesic rats. Pharmacol Biochem Behav 55: 151-156, 1996.

17. Zhao XP, Wang S and Xia YH: Effects of calcitonin injected into various brain areas on pain threshold and $\mathrm{Ca} 2+$ in rats. Zhongguo Yao Li Xue Bao 17: 218-220, 1996.

18. Ceserani R, Colombo M, Olgiati VR and Pecile A: Calcitonin and prostaglandin system. Life Sci 25: 1851, 1979.

19. Akgul C, Vural P and Canbaz M: Impact of calcitonin on urinary excretion of prostaglandins during menopause. Gynecol Obstet Invest 46: 199-201, 1998.

20. Stock JL and Coderre JA: Calcitonin enhances production of prostaglandins by stimulated human monocytes. Prostaglandins 27: 771-779, 1984.

21. Laurian L, Oberman Z, Graf E, Gilad S, Hoerer E and Simantov R: Calcitonin induced increase in ACTH, beta-endorphin and cortisol secretion. Horm Metab Res 18: 268-271, 1986.

22. Fabbri A, Santoro C, Moretti C, et al: The analgesic effect of calcitonin in humans: studies on the role of opioid peptides. Int J Clin Pharmacol Ther Toxicol 19: 509-511, 1981.

23. Fraioli F and Fabbri CR: Neurocalcitonin: analgesic action and autoradiographic distribution of the receptors in rat brain. $\mathbf{J}$ Endocrinol Invest 7: 177, 1984.

24. Sellami S and de Beaurepaire R: Medial diencephalic sites involved in calcitonin-induced hyperthermia and analgesia. Brain Res 616: 307-310, 1993.

25. Fischer JA, Tobler PH, Kaufmann M, et al: Calcitonin: regional distribution of the hormone and its binding sites in the human brain and pituitary. Proc Natl Acad Sci USA 78: 7801, 1981. 
26. Blanchard J, Menk E, Ramamurthy S and Hoffman J: Subarachnoid and epidural calcitonin in patients with pain due to metastatic cancer. J Pain Symptom Manage 5: 42-45, 1990.

27. Goicoechea C, Ormazabal MJ, Alfaro MJ and Martin MI: Effect of salmon-calcitonin on the analgesic effect of selective mu, delta and kappa opioid agonists in mice. Neurosci Lett 262: 25-28, 1999.
28. Knopp JA, Diner BM, Blitz M, Lyritis GP and Rowe BH: Calcitonin for treating acute pain of osteoporotic vertebral compression fractures: a systematic review of randomized, controlled trials. Osteoporos Int 16: 1281-1290, 2005.

29. Schiraldi GF, Soresi E, Locicero S, Harari S and Scoccia SL: Salmon calcitonin in cancer pain: comparison between two different treatment schedules. Int J Clin Pharmacol Ther Toxicol 25: 229-232, 1987. 